\title{
Multispectral Chiral Imaging with a Metalens
}

\section{Citation}

Khorasaninejad, M., W. T. Chen, A. Y. Zhu, J. Oh, R. C. Devlin, D. Rousso, and F. Capasso. 2016. "Multispectral Chiral Imaging with a Metalens." Nano Letters 16 (7): 4595-4600. https:// doi.org/10.1021/acs. nanolett.6b01897.

\section{Permanent link}

http://nrs.harvard.edu/urn-3:HUL.InstRepos:41371323

\section{Terms of Use}

This article was downloaded from Harvard University's DASH repository, WARNING: This file should NOT have been available for downloading from Harvard University's DASH repository.

\section{Share Your Story}

The Harvard community has made this article openly available.

Please share how this access benefits you. Submit a story.

Accessibility 


\title{
Multispectral Chiral Imaging with a Metalens
}

\author{
M. Khorasaninejad, ${ }^{*}{ }^{\dagger}$ W. T. Chen, ${ }^{\dagger}$ A. Y. Zhu, ${ }^{\dagger}{ }^{\prime}$. Oh, ${ }^{\dagger, \dagger}$ R. C. Devlin, ${ }^{\dagger}$ D. Rousso, ${ }^{\dagger, \dagger}$ and F. Capasso ${ }^{*}, \dagger$ \\ ${ }^{\dagger}$ Harvard John A. Paulson School of Engineering and Applied Sciences, Harvard University, Cambridge, Massachusetts 02138, United \\ States \\ ${ }^{\ddagger}$ University of Waterloo, Waterloo Ontario N2L 3G1, Canada
}

\section{Supporting Information}

\begin{abstract}
The vast majority of biologically active compounds, ranging from amino acids to essential nutrients such as glucose, possess intrinsic handedness. This in turn gives rise to chiral optical properties that provide a basis for detecting and quantifying enantio-specific concentrations of these molecules. However, traditional chiroptical spectroscopy and imaging techniques require cascading of multiple optical components in sophisticated setups. Here, we present a planar lens with an engineered dispersive response, which simultaneously forms two images with opposite helicity of an object within the same field-of-view. In this way, chiroptical properties can be probed across the visible spectrum using only the lens and a camera without the addition of polarizers

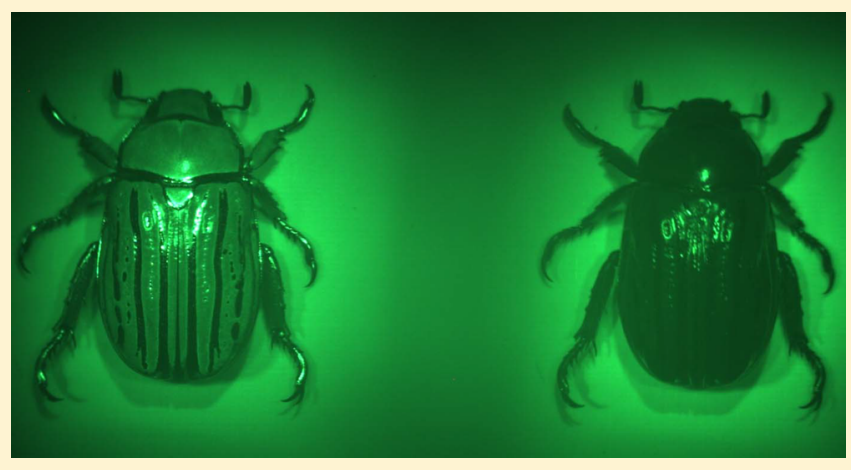
or dispersive optical devices. We map the circular dichroism of the exoskeleton of a chiral beetle, Chrysina gloriosa, which is known to exhibit high reflectivity of left-circularly polarized light, with high spatial resolution limited by the numerical aperture of the planar lens. Our results demonstrate the potential of metasurfaces in realizing a compact and multifunctional device with unprecedented imaging capabilities.
\end{abstract}

KEYWORDS: Metasurface, off-axis meta-lens, chiral imaging, multispectral, dispersive elements

$\mathrm{O}$ bjects are visible because they scatter light. Decoding spectral and polarization properties of scattered light can reveal valuable information about texture, orientation, and even the constituent materials of an object. ${ }^{1}$ Although we can partially perceive spectral information as color, our eyes are blind to the polarization states. Modern polarization imaging systems require cascading lenses with several optical components such as beamsplitters, polarizers, and waveplates. $^{1-3}$ Polarization-resolved imaging and spectroscopy have versatile applications ranging from remote ${ }^{4}$ and environmental $^{5}$ sensing to biological studies. ${ }^{6}$ For example, resolving chirality is essential for extracting structural and functional properties of biochemical species at both molecular and bulk levels. ${ }^{6-8}$ Besides costly and sizable configurations, cascading optical components also reduces the image quality and spatial resolution. Moreover, adding spectral resolution capabilities to an imaging system mandates the use of a dispersive element, ${ }^{9}$ integrated filters, ${ }^{10}$ or multiple cameras ${ }^{11}$ leading to further complexity and an increase of the instrument size. Here, we demonstrate a multispectral chiral lens (MCHL) that integrates the functionality of polarization and dispersive optical components into one device. This ultrathin lens overcomes the limitations of bulk optics and simultaneously provides chiral and spectral information across the visible spectrum without the requirement of additional optical components. The device can be utilized for both high-quality imaging and spectroscopy of bulk and microscopic scale specimens where the spatial resolution is limited by the numerical aperture (NA) of the MCHL.

Our MCHL utilizes a metasurface to achieve chiral imaging. Metasurfaces $^{12}$ offer the potential to incorporate unprecedented functionalities into an optically thin layer via a singlestep lithographic process. In recent years, it has been shown that they can control the optical properties of incident light such as intensity distribution, ${ }^{13,14}$ propagation direction, ${ }^{15-17}$ and polarization ${ }^{16,18-26}$ while bypassing the size and space requirements of conventional refractive optical devices. Although it has been shown that plasmonic ${ }^{27-34}$ and dielectric $^{19-21}$ metasurface-based lenses can distinguish the helicity of light, to the best of our knowledge there has been no demonstration of metasurfaces achieving chiral imaging.

The building blocks of the MCHL are comprised of two nanofins shown in blue and green in Figure 1a. The required phase for focusing light is imparted based on the Pancharatnam-Berry phase ${ }^{35,36}$ via rotation of nanofins (Figure $1 \mathrm{~b}$ ). The focusing efficiency is maximized when each nanofin acts as a half-wave plate, that is, it converts circularly polarized input light into transmitted light with opposite helicity. ${ }^{18-20,27}$ This is accomplished by suitably designing the nanofin dimensions

Received: May 10, 2016

Revised: June 5, 2016

Published: June 7, 2016 

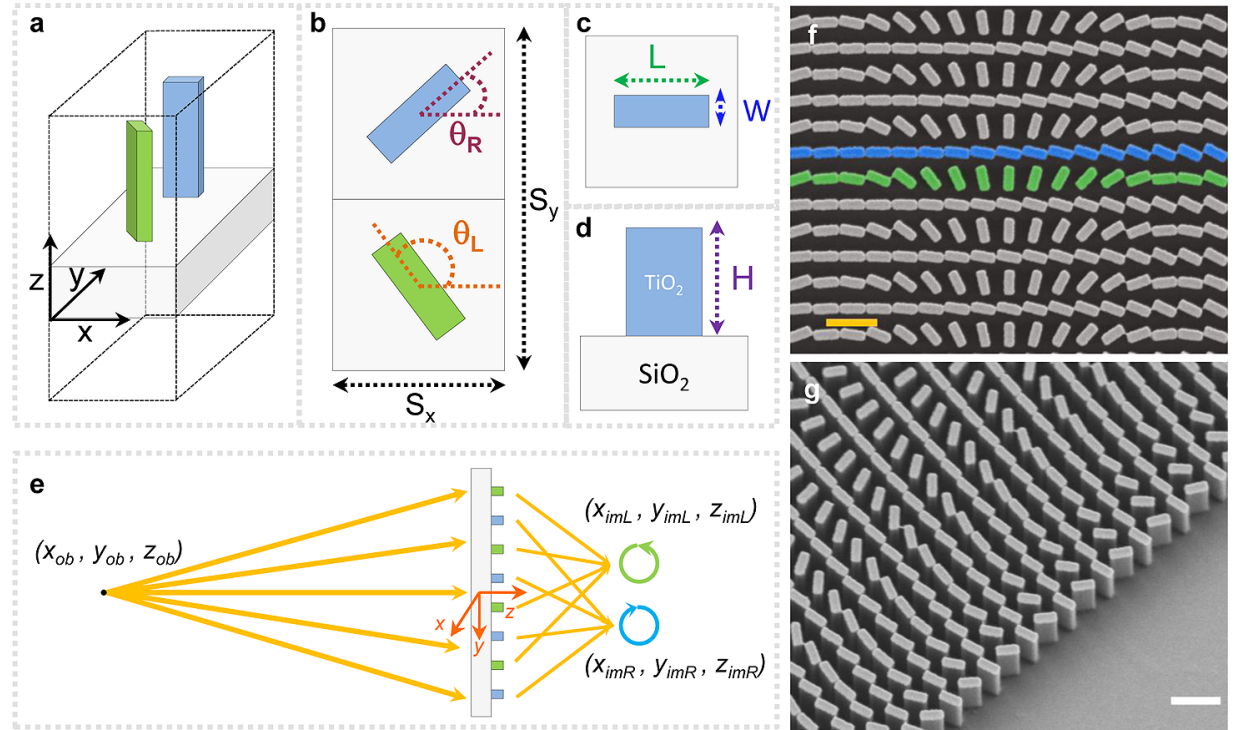

Figure 1. Design and fabrication of the multispectral chiral lens (MCHL). (a) The building block of the MCHL consists of two nanofins on a glass substrate. (b) Top-view of the building block where $S_{x}=300 \mathrm{~nm}$ and $S_{y}=600 \mathrm{~nm}$. The blue and green nanofins impart the required phase profile required to focus RCP light and LCP light, respectively. Phase realization is based on the geometric phase by means of nanofin rotation. (c) Topview of a nanofin denoting its length $L=250 \mathrm{~nm}$ and width $W=80 \mathrm{~nm}$. (d) Side-view of a nanofin showing its height $H=600 \mathrm{~nm}$. (e) Schematic diagram illustrating the imaging principle of the MCHL where LCP and RCP light from the same object at coordinates $\left(x_{\mathrm{ob}}=0, y_{\mathrm{ob}}=0, z_{\mathrm{ob}}=-18\right.$ $\mathrm{cm})$ are focused into two spots, $\left(x_{\mathrm{imL}}, y_{\mathrm{imL}}, z_{\mathrm{imL}}\right)$ and $\left(x_{\mathrm{imR}}, y_{\mathrm{imR}}, z_{\mathrm{imR}}\right)$, respectively. Spiral arrows indicate helicity of incident light. Coordinates of these spots are wavelength dependent due to the dispersive design of the MCHL. For the design wavelength of $530 \mathrm{~nm}$ these values are $x_{\mathrm{imL}}=-2.34$ $\mathrm{mm}, y_{\mathrm{imL}}=0.184 \mathrm{~mm}, z_{\mathrm{imL}}=29.90 \mathrm{~mm}, x_{\mathrm{imR}}=2.34 \mathrm{~mm}, y_{\mathrm{imR}}=0.184 \mathrm{~mm}$, and $z_{\mathrm{imR}}=29.90 \mathrm{~mm}$. (f) Top-view SEM image of the fabricated MCHL. Scale bar: $600 \mathrm{~nm}$. The two interlaced arrays of nanofins are false-colored. $(\mathrm{g})$ Side-view SEM image of a portion of the MCHL showing the highaspect-ratio $\mathrm{TiO}_{2}$ nanofins on a glass substrate. The MCHL has a diameter of $3 \mathrm{~mm}$. Scale bar: $600 \mathrm{~nm}$.

(Figure 1c,d): width $W$, length $L$, and height $H$. The schematic diagram of the MCHL, consisting of two interlaced arrays of blue and green nanofins on a glass substrate, is shown in Figure 1e. While the nanofins indicated by the color blue (located at $\left.\left(x_{R}, y_{R}, z_{R}\right)\right)$ impart the required phase profile for focusing incident right-circularly polarized (RCP) light, the nanofins in green (located at $\left.\left(x_{\mathrm{L}}, y_{\mathrm{L}}, z_{\mathrm{L}}\right)\right)$ focus left-circularly polarized (LCP) light. For an object located at $\left(x_{\mathrm{ob}}, y_{\mathrm{ob}}, z_{\mathrm{ob}}\right)$ the MCHL forms two side-by-side images with opposite helicity. The RCP image of the object is formed at $\left(x_{\mathrm{imR}}, y_{\mathrm{imR}}, z_{\mathrm{imR}}\right)$ and the LCP image at $\left(x_{\mathrm{imL}}, y_{\mathrm{imL}}, z_{\mathrm{imL}}\right)$.

In order to design the MCHL, we assume the object to be a point source with linear polarization, which is equivalent to a superposition of RCP and LCP components. First, we consider the RCP component and set the center of the MCHL $(x=0, y$ $=0, z=0)$ as the reference point. The phase delay $\varphi_{\mathrm{d}}$ due to the difference in the optical path from the object to image through the nanofin at position $\left(x_{R}, y_{R}, z_{R}\right)$ and the path through the reference point can be written as

$$
\varphi_{\mathrm{d}}\left(x_{\mathrm{R}}, y_{\mathrm{R}}, z_{\mathrm{R}}\right)=\frac{2 \pi}{\lambda_{\mathrm{d}}}\left(\Delta D_{\mathrm{ob}}+\Delta D_{\mathrm{im}}-f\right)
$$

where

$$
\begin{aligned}
& \Delta D_{\mathrm{ob}}=\sqrt{\left(x_{\mathrm{R}}-x_{\mathrm{ob}}\right)^{2}+\left(y_{\mathrm{R}}-y_{\mathrm{ob}}\right)^{2}+\left(z_{\mathrm{R}}-z_{\mathrm{ob}}\right)^{2}} \\
& \Delta D_{\mathrm{im}}=\sqrt{\left(x_{\mathrm{R}}-x_{\mathrm{imR}}\right)^{2}+\left(y_{\mathrm{R}}-y_{\mathrm{imR}}\right)^{2}+\left(z_{\mathrm{R}}-z_{\mathrm{imR}}\right)^{2}} \\
& f=f_{1}+f_{2} \\
& f_{1}={\sqrt{x_{\mathrm{ob}}{ }^{2}+y_{\mathrm{ob}}{ }^{2}+{z_{\mathrm{ob}}}^{2}}}
\end{aligned}
$$

$$
f_{2}=\sqrt{x_{\mathrm{imR}}^{2}+y_{\mathrm{imR}}^{2}+z_{\mathrm{imR}}^{2}}
$$

and $\lambda_{\mathrm{d}}=530 \mathrm{~nm}$ is the design wavelength. The reference optical path denoted by $f$ is the distance from the object to the reference point $\left(f_{1}=18 \mathrm{~cm}\right)$ plus the distance from the reference point to the image $\left(f_{2}=3 \mathrm{~cm}\right) . \Delta D_{\mathrm{ob}}$ and $\Delta D_{\text {im }}$ are distances between the nanofin at $\left(x_{\mathrm{R}}, y_{\mathrm{R}}, z_{\mathrm{R}}\right)$ and the object/ image, respectively. In order to form an image, this phase difference $\varphi_{\mathrm{d}}\left(x_{\mathrm{R}}, y_{\mathrm{R}}, z_{\mathrm{R}}\right)$ must be compensated by each nanofin. ${ }^{18-20}$ This is accomplished by rotating the nanofins by an angle $\theta_{\mathrm{R}}\left(x_{\mathrm{R}}, y_{\mathrm{R}}, z_{\mathrm{R}}\right)=-\frac{\varphi_{\mathrm{d}}\left(x_{\mathrm{R}}, y_{\mathrm{R}}, z_{\mathrm{R}}\right)}{2}$. A similar derivation is also valid for the LCP component of the point source except different arrays of nanofins located at the position $\left(x_{\mathrm{L}}, y_{\mathrm{L}}, z_{\mathrm{L}}\right)$ compensate the optical phase difference such that the image is formed at $\left(x_{\mathrm{imL}}, y_{\mathrm{imL}}, z_{\mathrm{imL}}\right)$. For LCP, the nanofins are rotated in the opposite direction with $\theta_{\mathrm{L}}\left(x_{\mathrm{L}}, y_{\mathrm{L}}, z_{\mathrm{L}}\right)=\frac{\varphi_{\mathrm{d}}\left(x_{\mathrm{L}}, y_{\mathrm{L}}, z_{\mathrm{L}}\right)}{2}$. We do not consider the effect of the glass substrate in our lens design. Theoretically this results in slight aberrations (Figure S1) in our case; however as we will show later this effect is negligible. The MCHL is fabricated by the method described in ref 37. Scanning electron microscope (SEM) images of the fabricated lens are shown in Figure 1f,g. High-aspect-ratio nanofins with negligible surface roughness are achieved, which is a characteristic of the atomic layer deposition of $\mathrm{TiO}_{2} \cdot{ }^{37}$ Recently, we reported high NA meta-objectives at visible wavelengths, suitable for subwavelength resolution microscopy using this fabrication process. ${ }^{38}$ However, they are unable to image both helicities of light simultaneously, which make them unfeasible for chiral bioimaging.

We analyzed the theoretical focusing characteristics of the lens using ray-tracing techniques by applying the phase gradient 

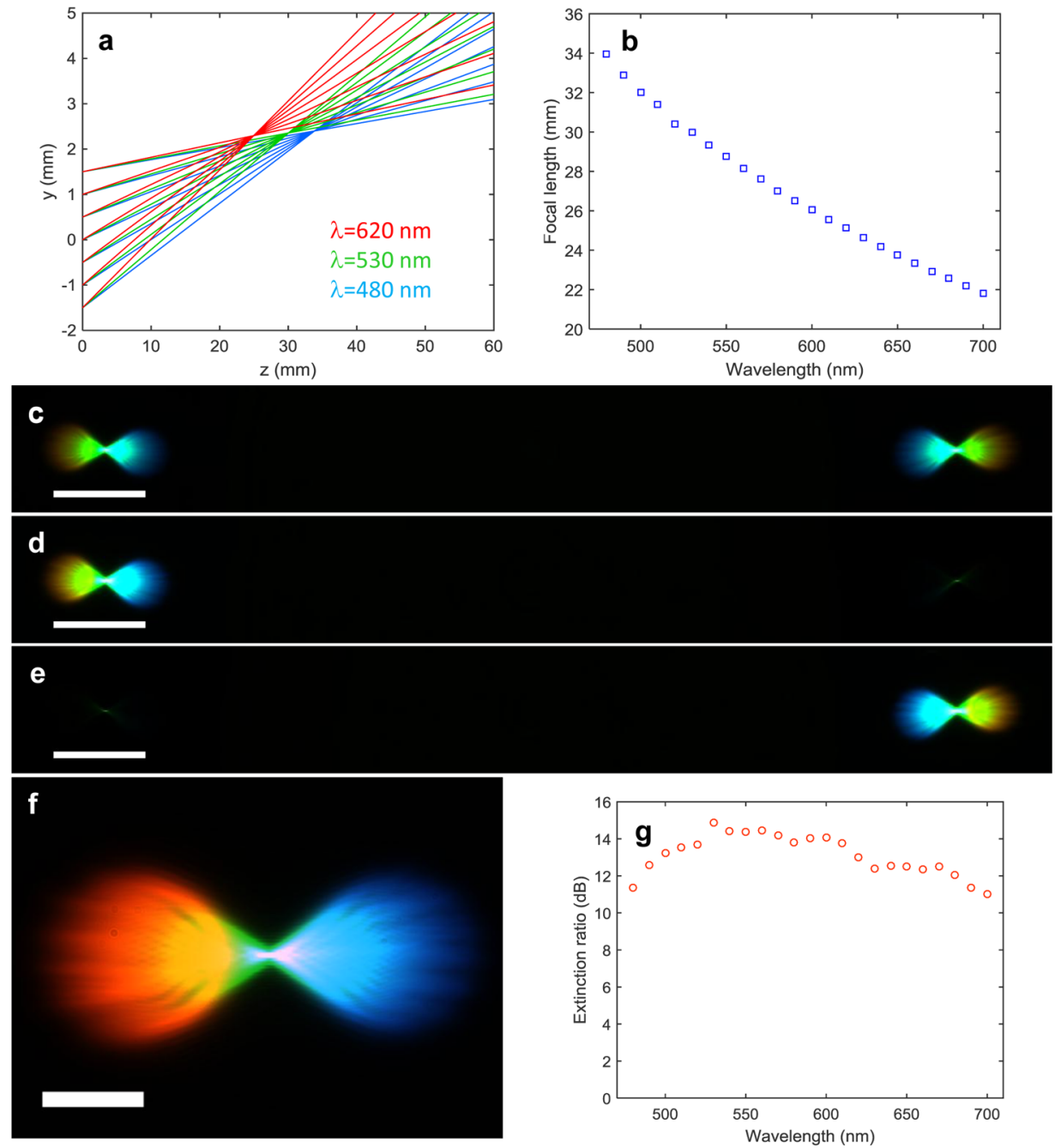

Figure 2. Characterization of the multispectral chiral lens (MCHL). (a) Simulation results using ray-tracing methods show the MCHL focusing incident light from a point source into a focal spot. The rays in this figure begin after passing through the MCHL. The full simulation domain is shown in Figure S2. The MCHL focuses different wavelengths into spatially separated spots enabling multispectral imaging. (b) Measured focal length as a function of wavelength. Here, focal length is defined as the distance from the focal spot to the center of the MCHL $(x=0, y=0, z=0)$. (c) Two images formed by the MCHL into the field-of-view of a color camera. The object was the facet of a single mode fiber emitting broadband light linearly polarized by a polarizer. The bandwidth is $100 \mathrm{~nm}$ centered at a wavelength of $550 \mathrm{~nm}$. (d) Images formed by the MCHL when the polarization of the emitting light is set to left-circularly polarized by adding a quarter-wave plate after the polarizer. (e) Images formed by the MCHL when the emitting light is switched to right-circularly polarized by adjusting the quarter-wave plate appropriately. The gain and exposure time of the camera were fixed for panels $\mathrm{c}-\mathrm{e}$. Also the camera was saturated at the focal point making it possible to visualize the out of focus colors. For panels $\mathrm{c}-\mathrm{e}$, the scale bar is $0.5 \mathrm{~mm}$. (f) Magnified view of the left image in panel c. Scale bar: $0.150 \mathrm{~mm}$. (g) Measured extinction ratio of the MCHL as a function of wavelength.

introduced by the nanofins onto the incident rays. Figure $2 \mathrm{a}$ and Figure S2 show that the MCHL focuses all incident rays emanating from a point source into a focal point. Here, the position of the focus shifts by changing the wavelength as shown in Figure $2 b$. This is associated with the off-axis focusing of the MCHL as well as the intrinsic dispersion of metasurfacebased lenses (Figure S3 and Note 1). We experimentally observed this effect by imaging the facet of a single mode fiber. Figure $2 c$ shows how the MCHL simultaneously focuses and disperses a broadband beam emitted from the fiber and forms a rainbow-like image. The beam had a center wavelength of 550 $\mathrm{nm}$ with a bandwidth of $100 \mathrm{~nm}$ (Methods). Because of the wavelength dependence of the focal length, the MCHL focuses green light while the other colors are out of focus. This effect is more clearly seen in the magnified image in Figure $2 f$. However, by adjusting the camera position, one can selectively focus onto other wavelengths (Figure S4 and Movie 1).

Figure $2 \mathrm{c}$ also shows that for a linearly polarized light source there are two identical images within the camera's field-of-view, formed by the LCP and RCP components of the incident light. It is notable that according to the phase profile of our MCHL, the array of nanofins that focuses one type of helicity will simultaneously diverge the light of opposite helicity (virtual image), which contributes to the background noise. However, this effect is negligible as shown in Figure $2 c$ and Figure S5 where there are no higher orders or noticeable background noise. Next, the chiral response of the MCHL is examined by setting the polarization state of the incident light to LCP. As shown in Figure 2d and Movie 2, the right image disappears with a corresponding increase in intensity of the left image. The opposite effect was observed by switching the incident helicity 

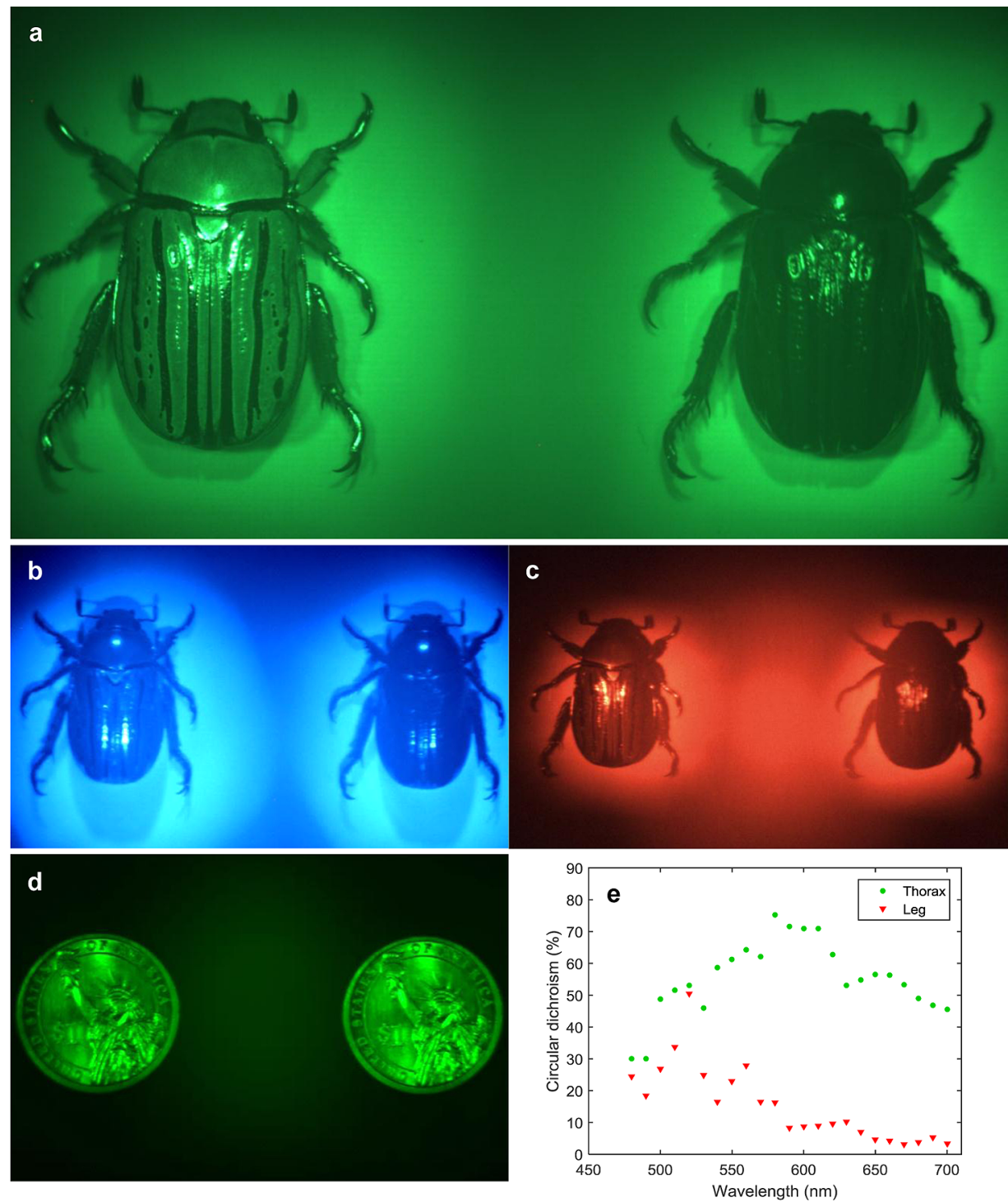

Figure 3. Imaging with the multispectral chiral lens (MCHL). (a) The MCHL forms two images of the beetle, Chrysina gloriosa, on the color camera. The left image is formed by focusing left-circularly polarized light reflected from the beetle and the right image is from right-circularly polarized light reflected from the beetle. Illumination was provided by green LEDs paired with $10 \mathrm{~nm}$ bandpass filter centered at $532 \mathrm{~nm}$. (b,c) Images formed by the MCHL under (b) blue (10 nm bandpass filter centered at $488 \mathrm{~nm}$ ) and (c) red LED illumination (10 nm bandpass filter centered at $620 \mathrm{~nm})$. (d) Images of a one-dollar coin under green LED illumination (LED was paired with $10 \mathrm{~nm}$ bandpass filter centered at $532 \mathrm{~nm}$ ). (e) Circular dichroism from two different parts of the beetle as a function of wavelength.

(Figure 2e). We quantified this chiral response by measuring the extinction ratio (ER) using the method described by Azzam et al. ${ }^{39}$ In the latter, ER is defined as the ratio of optical power of the right (left) image to the left (right) image under RCP (LCP) illumination. Figure $2 \mathrm{~g}$ plots the values of the ER across the visible spectrum. A high ER of $15 \mathrm{~dB}$ together with the engineered dispersive properties of the MCHL makes it possible to perform multispectral chiroptical imaging.

For chiral imaging across the visible range, we chose a large biological specimen, Chrysina gloriosa ${ }^{40,41}$ which is a beetle that naturally exhibits strong circular dichroism (CD) at green wavelengths. The imaging setup is shown in Figure S6 and discussed in the Methods. Figure 3a shows that the MCHL simultaneously forms two images of the beetle on the camera chip with opposite helicity. The very strong CD of the beetle is evident by comparing images of the beetle on the right- and left-handed side, as the beetle's exoskeleton strongly reflects
LCP light while absorbing more RCP light. ${ }^{40,41}$ Images of the beetle under blue and red LED illumination are shown in Figure 3 panels $\mathrm{b}$ and $\mathrm{c}$, respectively. The slight background noise partially comes from the lower efficiency of the MCHL (Figure S7) at red and blue wavelengths in addition to the lower reflectivity of the beetle in this region. ${ }^{41} \mathrm{We}$ also imaged an achiral object, a one-dollar coin. As expected, Figure $3 \mathrm{~d}$ shows two nearly identical images formed by the MCHL. Finally, we measured the $\mathrm{CD}$ of different body parts of the beetle using the measurement configuration shown in Figure S8. First, we focused laser light onto the beetle's thorax. The focal spot has a size of $\sim 100 \mu \mathrm{m}$ to avoid sample damage. The scattered light is captured by the MCHL, which forms two spots with different intensity corresponding to the two circularly polarized states $\left(I_{\mathrm{R}}\right.$ and $\left.I_{\mathrm{L}}\right)$ on the camera. The CD is calculated by $\frac{I_{L}-I_{R}}{I_{L}+I_{R}}$ while sweeping the wavelength. This measurement was subsequently repeated on its leg. The results 
in Figure 3e show large CD that peaks around the green region of the spectrum. In our approach, we used the MCHL to image a chiral beetle but it can be more generally utilized to image a wide range of chiral objects. Ultimately, the spatial resolution is determined by the NA of the MCHL. Here, in order to provide a large field-of-view to image the entire beetle, we chose a large focal length resulting in a low NA $=0.05 .{ }^{17}$ The image resolution can be readily improved via reducing the focal length or increasing the diameter of the MCHL.

In summary, we experimentally demonstrated, for the first time, chiral imaging capabilities and spatially resolved chiral spectroscopy with a single planar lens. Conventionally, this cannot be achieved using single optical components unless multiples of them are stacked together into a bulky system. The MCHL's engineered dispersive response also enables multispectral imaging; we measured the $\mathrm{CD}$ of a chiral object for a broad range of visible wavelengths. Providing multiple functionalities while retaining a planar and compact geometry highlights the many potential scientific and practical applications of our lens. We envision that the MCHL will find widespread uses in numerous fields ranging from biology and pharmacology to environmental and remote sensing.

Methods. Imaging with MCHL. For imaging the facet of the single mode fiber, we used the experimental setup shown in Figure S9. We placed a wire grid polarizer (Thorlabs WP25LUB) and quarter-waveplate (Thorlabs AQWP05M-600) between the fiber and the MCHL to control the polarization state of the incident beam. The fiber was connected to a tunable laser (SuperK NKT photonics). Images were captured by a color camera (Point Gray, GX-FW-28S5C-C) located on the side of the MCHL opposite to the fiber. For imaging the beetle, we replaced the fiber with the beetle (Figure S6) and illumination was provided by LEDs paired with proper bandpass filters with a $10 \mathrm{~nm}$ bandwidth to reduce chromatic aberration effects. For CD measurements, illumination light was a tunable laser (SuperK NKT photonics) with a bandwidth of $10 \mathrm{~nm}$ focused on the beetle. The focal spot has a full width at half-maximum of $\sim 100 \mu \mathrm{m}$.

\section{ASSOCIATED CONTENT}

\section{S Supporting Information}

The Supporting Information is available free of charge on the ACS Publications website at DOI: 10.1021/acs.nanolett.6b01897.

Figures $\mathrm{S} 1-\mathrm{S} 9$ and Note 1 (Engineering the dispersive response of multispectral chiral lens (MCHL)). (PDF) Movie showing how MCHL simultaneously focuses and disperses a broadband beam emitted from the fiber and forms a rainbow-like image. (AVI)

Movie showing how MCHL focuses incident light based on its helicity and forms two images with opposite helicity in the same field-of-view of a camera. (AVI)

\section{AUTHOR INFORMATION}

\section{Corresponding Authors}

*E-mail: khorasani@seas.harvard.edu.

*E-mail: capasso@seas.harvard.edu.

\section{Notes}

The authors declare no competing financial interest.

\section{ACKNOWLEDGMENTS}

This work was supported in part by the Air Force Office of Scientific Research (MURI, Grants FA9550-14-1-0389 and FA9550-16-1-0156), Charles Stark Draper Laboratory, Inc. (SC001-0000000959) and Thorlabs Inc. W.T.C. acknowledges postdoctoral fellowship support from the Ministry of Science and Technology, Taiwan (104-2917-I-564-058). A.Y.Z. thanks Harvard SEAS and A*STAR Singapore under the National Science Scholarship scheme. R.C.D. is supported by a Charles Stark Draper Fellowship. This work was performed in part at the Center for Nanoscale Systems (CNS), a member of the National Nanotechnology Coordinated Infrastructure (NNCI), which is supported by the National Science Foundation under NSF award no. 1541959. CNS is a part of Harvard University. We thank $\mathrm{E}$. $\mathrm{Hu}$ for the supercontinuum laser (NKT "SuperK").

\section{REFERENCES}

(1) Azzam, R. M.; Bashara, N. M. Ellipsometry and polarized light; Elsevier Science Publishing Co., Inc.: New York, 1987.

(2) Groner, W.; Winkelman, J. W.; Harris, A. G.; Ince, C.; Bouma, G. J.; Messmer, K.; Nadeau, R. G. Nat. Med. 1999, 5 (10), 1209-1212.

(3) Tyo, J.; Rowe, M.; Pugh, E.; Engheta, N. Appl. Opt. 1996, 35 (11), 1855-1870.

(4) Deuzé, J.; Bréon, F.; Devaux, C.; Goloub, P.; Herman, M.; Lafrance, B.; Maignan, F.; Marchand, A.; Nadal, F.; Perry, G. J. Geophys. R.: Atmos. 2001, 106 (D5), 4913-4926.

(5) Knyazikhin, Y.; Schull, M. A.; Stenberg, P.; Mõttus, M.; Rautiainen, M.; Yang, Y.; Marshak, A.; Carmona, P. L.; Kaufmann, R. K.; Lewis, P. Proc. Natl. Acad. Sci. U.S.A. 2013, 110 (3), E185E192.

(6) McNichols, R. J.; Cote, G. L. J. Biomed. Opt. 2000, 5 (1), 5-16.

(7) Berova, N.; Nakanishi, K. Circular dichroism: principles and applications; John Wiley \& Sons: New York, 2000.

(8) Srinivasarao, M. Chem. Rev. 1999, 99 (7), 1935-1962.

(9) Fischer, C.; Kakoulli, I. Stud. Conserv. 2006, 51 (sup1), 3-16.

(10) Yamaguchi, M.; Teraji, T.; Ohsawa, K.; Uchiyama, T.; Motomura, H.; Murakami, Y.; Ohyama, N. In Color image reproduction based on multispectral and multiprimary imaging: experimental evaluation; International Society for Optics and Photonics: Bristol, U.K., 2002; pp 15-26.

(11) Everitt, J.; Escobar, D.; Cavazos, I.; Noriega, J.; Davis, M. Remote sensing of environment 1995, 54 (3), 333-337.

(12) Yu, N.; Capasso, F. Nat. Mater. 2014, 13 (2), 139-150.

(13) Yu, N.; Genevet, P.; Kats, M. A.; Aieta, F.; Tetienne, J.-P.; Capasso, F.; Gaburro, Z. Science 2011, 334 (6054), 333-337.

(14) Khorasaninejad, M.; Aieta, F.; Kanhaiya, P.; Kats, M. A.; Genevet, P.; Rousso, D.; Capasso, F. Nano Lett. 2015, 15 (8), 53585362.

(15) Ni, X.; Emani, N. K.; Kildishev, A. V.; Boltasseva, A.; Shalaev, V. M. Science 2012, 335 (6067), 427-427.

(16) Khorasaninejad, M.; Zhu, W.; Crozier, K. Optica 2015, 2 (4), $376-382$.

(17) Khorasaninejad, M.; Chen, W. T.; Oh, J.; Capasso, F. Nano Lett. 2016, 16, 3732.

(18) Bomzon, Z. e.; Biener, G.; Kleiner, V.; Hasman, E. Opt. Lett. 2002, 27 (5), 285-287.

(19) Lin, D.; Fan, P.; Hasman, E.; Brongersma, M. L. Science 2014, 345 (6194), 298-302.

(20) Khorasaninejad, M.; Crozier, K. B. Nat. Commun. 2014, 5, 5386.

(21) Arbabi, A.; Horie, Y.; Bagheri, M.; Faraon, A. Nat. Nanotechnol. 2015, 10, 937-943.

(22) Cong, L.; Srivastava, Y. K.; Singh, R. Adv. Opt. Mater. 2016, DOI: 10.1002/adom.201500681.

(23) Khorasaninejad, M.; Ambrosio, A.; Kanhaiya, P.; Capasso, F. Sci. Adv. 2016, 2 (5), e1501258. 
(24) Plum, E.; Fedotov, V.; Zheludev, N. J. Opt. A: Pure Appl. Opt.

2009, 11 (7), 074009.

(25) Plum, E.; Liu, X.-X.; Fedotov, V.; Chen, Y.; Tsai, D.; Zheludev, N. Phys. Rev. Lett. 2009, 102 (11), 113902.

(26) Huang, L.; Chen, X.; Mühlenbernd, H.; Zhang, H.; Chen, S.; Bai, B.; Tan, Q.; Jin, G.; Cheah, K.-W.; Qiu, C.-W. Nat. Commun. 2013, 4, 2807.

(27) Zheng, G.; Mühlenbernd, H.; Kenney, M.; Li, G.; Zentgraf, T.; Zhang, S. Nat. Nanotechnol. 2015, 10 (4), 308-312.

(28) Chen, X.; Huang, L.; Mühlenbernd, H.; Li, G.; Bai, B.; Tan, Q.; Jin, G.; Qiu, C.-W.; Zhang, S.; Zentgraf, T. Nat. Commun. 2012, 3, 1198.

(29) Zhao, Y.; Belkin, M.; Alù, A. Nat. Commun. 2012, 3, 870.

(30) Gansel, J. K.; Thiel, M.; Rill, M. S.; Decker, M.; Bade, K.; Saile, V.; von Freymann, G.; Linden, S.; Wegener, M. Science 2009, 325 (5947), 1513-1515.

(31) Papakostas, A.; Potts, A.; Bagnall, D.; Prosvirnin, S.; Coles, H.; Zheludev, N. Phys. Rev. Lett. 2003, 90 (10), 107404.

(32) Wu, C.; Arju, N.; Kelp, G.; Fan, J. A.; Dominguez, J.; Gonzales, E.; Tutuc, E.; Brener, I.; Shvets, G. Nat. Commun. 2014, 5, 3892.

(33) Fedotov, V.; Mladyonov, P.; Prosvirnin, S.; Rogacheva, A.; Chen, Y.; Zheludev, N. Phys. Rev. Lett. 2006, 97 (16), 167401.

(34) Yin, X.; Ye, Z.; Rho, J.; Wang, Y.; Zhang, X. Science 2013, 339 (6126), 1405-1407.

(35) Pancharatnam, S. In Generalized theory of interference and its applicationsl; Proceedings of the Indian Academy of Sciences-Section A,; Springer: New York, 1956; pp 398-417.

(36) Berry, M. V. J. Mod. Opt. 1987, 34 (11), 1401-1407.

(37) Devlin, R. C.; Khorasaninejad, M.; Chen, W. T.; Oh, J.; Capasso, F. 2016, arXiv: 1603.02735.

(38) Khorasaninejad, M.; Chen, W.-T.; Devlin, R. C.; Oh, J.; Zhu, A.; Capasso, F. Science 2016, 352 (6290), 1190-1194.

(39) Azzam, R.; Lopez, A. G. J. Opt. Soc. Am. A 1989, 6 (10), 15131521.

(40) Sharma, V.; Crne, M.; Park, J. O.; Srinivasarao, M. Science 2009, 325 (5939), 449-451.

(41) Goldstein, D. H. Appl. Opt. 2006, 45 (30), 7944-7950. 